\title{
RELAÇĀO DE NOMES E CARACTERÍSTICAS DE ESTRATÉGIAS DE COLETA DE DADOS
}

Silvia Helena de Bortoli Cassiani*

CASSIANI, S.H. de B. Relação de nomes e características de estratégias de coleta de dados. Rev. Esc. Enf. USP, Sāo Paulo, 23(3):345-357, dez. 1989.

Realizado levantamento em três revistas de enfermagem americana e teses nacionais sobre estudos que trataram do desenvolvimento de estratégias de coleta de dados. Resultou em 22 estratégias, sendo duas destas analisadas no estudo. As restantes são apresentadas sob a forma de lista dividida entre nacionais $e$ internacionais.

UNITERMOS: Pesquisa em enfermagem. Coleta de dados.

\section{INTRODUÇẢO}

Embora seja de consenso entre os pesquisadores, é de se enfatizar que todo processo científico prescinde de uma etapa de coleta de dados.

Os dados são gerados a partir da utilização de uma estratégia de coleta, e a seguir analisados para que resultem em conhecimentos.

A literatura possui terminologia variada a respeito de meios de coletar os dados. São encontrados os termos: métodos de coleta de dados (NOTTER, 1978; BUCHANAN, 1981 e POLIT \& HUNGLER, 1983), instrumentos de coletar dados (WILLIAMSON, 1981; NEVES, 1982), instrumentos de pesquisa (PINTO, 1979; GONÇALVES, 1981; UBEDA, 1986) e mais recentemente tem-se empregado o termo estratégia de coleta de dados (E.C.D.) (LYNN, 1985) e que será o utilizado neste estudo.

TREECE \& TREECE (1977) explicam que há quatro técnicas básicas para obter dados: questionários e entrevistas, registros e técnicas de observação e experimentação, sendo que, cada uma dessas abordagens contém numerosos tipos de instrumentos tão inovativos quanto a capacidade do pesquisador admitir.

Assim como estes, NOTTER (1979), NEVES (1982) e POLIT \& HUNGLER (1983) reconhecem que as estratégias mais utilizadas nas pesquisas em enfermagem são: entrevistas, questionários, técnicas de observação e revisão de re-

* Professor Assistente do Departamento de Enfermagem Geral e Especializada da EERP-USP. 
gistros. Notadamente, verificamos a carência de utilização de outras estratégias como: escalas, técnica de Delphi e outras metodologias.

Este investigador em um estudo que buscou identificar quais eram as estratégias utilizadas nas teses de doutorado, dissertações de mestrado e artigos da Revista Brasileira de Enfermagem no período de 1977 a 1986, concluiu que em $88,6 \%$ das pesquisas analisadas, as estratégias mais utilizadas, foram: entrevista, questionário, formulário, medidas fisiológicas e observação sendo que a entrevista foi mais empregada nas dissertações de mestrado, enquanto que o questionário foi mais utilizado nas publicaçōes consultadas da Revista Brasileira de Enfermagem (CASSIANI, 1987).

A necessidade de melhoria dos instrumentos e técnicas de coleta de dados empregadas foi enfatizado no documento AVALIAÇĀO \& PERSPECTIVAS (1982) nāo explicando-nos, entretanto, o que seja essa "melhoria" pretendida.

Análises das estratégias de coleta de dados apresentadas na revista Nursing Research, no período de 1970 a 1978 , mostrou que existe uma tendência entre os pesquisadores americanos em utilizar instrumentos previamente desenvolvidos, o que reflete preocupação destes com aspectos referentes a validade e confiabilidade dos instrumentos (O’CONNELL, 1983).

Porém, segundo BROWN et alii (1984), apesar desta preocupação com estratégicas válidas e confiáveis, ainda constitui-se verdade que a maioria dos pesquisadores falham ao detalhar os testes e medidas de validade e confiabilidade empregadas.

Ainda o estudo que realizamos em 1987 , demonstrou que em $57,5 \%$ ds pesquisas analisadas, o investigador, não cita a utilizaçāo de procedimentos para evidenciarem a validade e confiabilidade das estratégias (CASSIANI, 1987). O que constitui uma evidência importante, se considerarmos a seguinte citação: "com a falta de confiabilidade (e validade) nas pesquisas em enfermagem, muitos dados, com os quais a enfermagem está tentando construir a ciência da enfermagem, não poderão ser considerados como tal. Os esforços da enfermagem para a credibilidade científica serão seriamente afetados à menos que a confiabilidade e portanto a qualidade das medidas usadas nas pesquisas sejam apontadas" (LYNN, 1985, p. 256).

Os pesquisadores quando da construção das estratégias de coleta dos dados devem evidenciar a validade e confiabilidade desta para aquela situação. Tal procedimento facilitará o trabalho de outros que poderão utilizar-se desta estratégia.

É aparente a facilidade que estratégias já desenvolvidas e validadas fornecem à comunidade científica, que desta forma não necessitará individualmente de desenvolver suas próprias estratégias, se aquelas após avaliação, se adequarem aos estudos propostos. 
Afirmamos porém que a grande maioria dos pesquisadores em enfermagem no Brasil continua a desenvolver, para cada estudo, a estratégia com que coletará os dados, sendo poucos os que utilizam estratégias previamente elaboradas (CASSIANI, 1987).

Temos observado a publicação de estudos, mais a nível internacional com a construçāo ou o desenvolvimento de estratégias, visando torná-los conhecido para que possam ser utilizados ou mesmo aperfeiçoado por outros investigadores.

Entretanto localizar uma estratégia apropriada para o estudo pretendido é algumas vezes difícil, a alternativa é então construir uma nova estratégia.

Sendo esta etapa de construção e adequação, um trabalho árduo, que exige uma certa experiência e consome tempo e dinheiro do investigador.

Conhecedora destas dificuldades, por nossa própria experiência com pesquisas, decidimos neste estudo por apresentar uma relação de nomes e características de estratégias de coleta de dados, visando mais uma vez divulgá-las para que possam ser empregadas nas investigações em enfermagem.

\section{OBJETIVOS}

É objetivo deste estudo levantar investigaçōes metodológicas que trataram do desenvolvimento de instrumentos e apresentar uma lista de nomes e características das estratégias elaboradas nestes trabalhos.

\section{METODOLOGIA}

\section{- População}

Consideramos como universo deste estudo as Revistas International Journal of Nursing Studies, Nursing Research e Research in Nursing and Health no periodo de 1980 a 1987. A revista Research in Nursing and Health foi consultada no período de 1983 a 1987. Foi incluído nessa população o banco de teses da Escola de Enfermagem de Ribeirão Preto-USP.

Estas revistas foram as escolhidas uma vez que são as que mais divulgam investigações metodológicas que tratam especificamente do desenvolvimento de instrumentos.

\section{- Material e Método}

As revistas e dissertações de mestrado e teses no período de 1980 a 1987 foram revistas e artigos e trabalhos metodológicos que descreviam o desenvolvimento de instrumentos foram extraídos.

Cada estudo foi analisado e as seguintes informações foram coletadas através 
de formulário: nome e objetivo do instrumento, referencial teórico, características do instrumento, nome e endereço do autor e a referência bibliográfica que continha o instrumento.

Vale ressaltar que o nome e endereço do autor foram coletados para que sejam contactuados e solicitado permissão quando da utilizaçāo da estratégia por eles desenvolvida.

A fim de assegurar a qualidade e confiabilidade do formulário proposto, submetê-lo a duas aplicações, ou seja, foram revistos três trabalhos com intervalo de uma semana entre as aplicações e os resultados obtidos foram comparados. Obtivemos uma concordância de $100 \%$.

\section{RESULTADOS E DISCUSSÃO}

Da análise dos periódicos e do banco de teses foram extraídos vinte e duas (22) estratégias de coleta de dados, sendo dezoito (18) estratégias de revistas internacionais e duas (2) do banco de teses analisado.

A listagem está apresentada em anexo.

Do total, dezoito (18) estratégias foram extraídas da revista Research in Nursing and Health, quatro (4) da Nursing Research e uma (1) da International Nursing Studies.

Destas estratégias, dezoito (18) apresentavam-se sob forma de escalas, duas (2) eram questionários, uma (1) observação e uma (1) entrevista estruturada.

Observamos que em todos estes, procedimentos relativos à validade e confiabilidade são descritos, alguns sugerem ao final do estudo que outros pesquisadores continuem a desenvolver o instrumento e suas diversas aplicaçōes.

Procedemos a análise de duas estratégias coletadas e que passaremos a comentar a seguir.

\section{INSTRUMENTO}

Interpersonal Conflict Scale

Objetivo:

Medir conflitos interpessoais entre os casais. Referencial teórico:

Derivado de trabalho de teóricos e pesquisadores sobre conflito familiar e relacionamento interpessoal. Cita principalmente o trabalho de MATHEWS e MIHANOVICH (1963)* como base referencial.

* MATHEWS, V.D.; MIHANOVICH, C.S. New orientations on marital adjustment. Marriage and Family
Living, 25: 300-34, 1963. 


\section{Características:}

O instrumento consiste de 90 ítens, divididos em nove categorias, sendo portanto, 10 ítens em cada categoria. As categorias são: concordância no pensamento, comunicação, discordância no pensamento, percepção do sentimento de outros, companheirismo, percepção do comportamento, satisfação emocional, segurança e reconhecimento.

$\mathrm{O}$ autor evidenciou a validade e confiabilidade do instrumento. A confiabilidade foi obtida através do método do teste-reteste, ou seja, o instrumento foi aplicado duas vezes e os escores foram comparados. A seguir usou-se o coeficiente de correlação produto-momento de Pearson. $O$ índice obtido variou de 0,84 a 0,95 , o que demonstra ser um instrumento de alto índice de confiabilidade*.

$\mathrm{O}$ método do teste-reteste pretende estimar a estabilidade do instrumento. Consiste na administração ao mesmo tempo a um grupo de indivíduos em duas ocasiōes diferentes e na comparação dos escores obtidos.

Porém uma vez que este método foi utilizado neste instrumento, apontaremos algumas desvantagens:

- As respostas da segunda administração, podem ser influenciadas pela da primeira administração, ou seja, os respondentes podem lembrar-se da sua primeira resposta e tentar dar a mesma resposta na segunda vez (NUNNALY, 1970).

- Os respondentes podem mudar com os resultados da primeira administração.

- Há possibilidade de mudanças genuínas terem ocorrido entre os períodos.

- Fatores pessoais podem influenciar mais na segunda medição.

Apesar destas desvantagens é um método eficiente, porém não deve ser utilizado em situaçōes em que o atributo pode mudar no período do teste-reteste. Neste cado o método estaria medindo as mudanças ocorridas nos indivíduos e não a estabilidade do instrumento.

No caso do constructo deste instrumento ser conflito interpessoal entre casais pode ter havido modificações nas relações entre a primeira e a segunda administração, e além disto a memória pode ter interferido tornando os escores nas duas administrações similares, o que não indicaria a realização deste método para estimar a confiabilidade do instrumento.

Seria mais adequado a verificação da consistência interna do instrumento através do Coeficiente Alpha de Cronbach, comparando os dois índices de confiabilidade obtidos.

\footnotetext{
* Na prática o coeficiente de confiabilidade varia de 0 a 1 . Quanto mais alto o coeficiente mais estável é a medida. Para alguns propósitos, o coeficiente acima de 0,70 é considerado satisfatório (POLIT \& HUNGLER, 1983). NUNNALLY (1970) indica que os melhores testes tem coeficientes de confiabilidade entre 0,80 e 0,95 .
} 
A validade de conteúdo foi obtida através da análise de juizes.

$O$ autor sugere ao final que estudos sejam realizados determinando a relação entre as respostas dos itens e o sexo do respondente. Indica que o desenvolvimento do instrumento será mais estudado.
Autor:
Carol Noll Hoskins
Division of Nursing
New York University
429 Shinikin Hall
Washington Square
New York, NY 1003.

Referências Bibliográficas:

Psychometries in Nursing Research: Construction of an interpersonal conflict scale. Research in Nursing and Health 4(2): 243-249, junho 1981.

Utilização para enfermagem: para aqueles interessados no estudo de temáticas referentes à família, o instrumento pode ser de grande utilização.

O segundo instrumento analisado foi "A semantic differential measure of attitude toward black american patients".

Objetivo: medir atitudes dos membros da enfermagem em relação aos negros.

Referencial teórico: $\mathrm{O}$ autor cita alguns estudos que lidam com a questāo, porém não descreve o referencial teórico que embasou a elaboração do instrumento.

Características: $\mathrm{O}$ instrumento consiste de 20 escalas sob a forma do diferencial semântico de Osgood, com adjetivos bipolares para estereótipos raciais.

A escala do diferencial semântico é apresentada ao respondente contendo um dado conceito e a seguir uma escala formada por sete intervalos tendo adjetivos bipolares em suas extremidades. $O$ respondente marca segundo sua percepção, 0 adjetivo correspondente aquele conceito em algum ponto da escala.

A confiabilidade das escalas do diferente semântico enfocará a reprodutibilidade dos escores em repetidas medições.

A estabilidade através do teste-reteste, pode ser determinada. A validade de conteúdo e a validade relacionada ao critério pode ser também evidenciada.

A confiabilidade desta escala segundo seu autor foi de 0,84 a validade de conteúdo foi descrita no estudo.

Utilização na enfermagem:

Pode ser utilizada em pesquisas para medir atitudes frente aos pacientes negros. 
Autor:

Barbara S. Morgan

College of Nursing

University of Rhode Island

Kingston. LI 02881

Referência:

A Semantic Differential Measure of Attitude toward black american patients. $R e$ search in Nursing and Health 17(3): 155-162, set. 1984.

\section{CONCLUSÕES}

Esperamos que a lista de estratégias de coleta de dados seja mais um auxílio aos pesquisadores em enfermagem. $O$ nome do autor que elaborou o instrumento, o endereço destes e a fonte bibliográfica estão apresentados com o intuito de facilitar o trabalho daqueles que desejarem utilizar uma destas estratégias em contactuar $o$ autor e requerer $o$ instrumento.

E finalizamos com a citação de POLIT \& HUNGLER (1983) quando afirmam que: "o pesquisador deve considerar a existência de medidas antes de embarcar num projeto para construir novas escalas. $O$ desenvolvimento de bons instrumentos é árduo e consome muito tempo. E uma vez que centenas de medidas estāo disponíveis, algum esforço deve ser gasto para identificar aqueles instrumentos que cobrem as variáveis de interesse" (p. 340).

CASSIANI, S.H. de H. List of names and characteristics of data collection strategies. Rev. Esc. Enf USP, São Paulo, 23(3):345-357, dez. 1989.

The study is a survey in three american nursing journals and national thesis in order to find studies on the development of data collection strategies. The results showed 22 strategies. Two of them were analysed in this study. The following strategies are presented in a list divided into international and national studies.

UNITERMS: Research in nursing. Data collection.

\section{REFERÊNCIAS BIBLIOGRÁFICAS}

1 AVALIAÇÃO \& perspectivas: ciências da saúde-enfermagem. Brasília, Secretaria de Planejamento, 1982. v. 2.

2 BuChanan, M.G. Methods of data collection. Assoc. Oper. Room Nurs. J., New York, 33(1): 137-149, ja. 1981.

3 BROWN, J.S.; TANNER, C.A.; PADDRICK, K.P. Nursing's search for scientific knowlledge. Nurs. Res., New York, 33(1): 26-32, Jan./Feb. 1984.

4 CASSIANI, S.H.B. A coleta de dados nas pesquisas em enfermagem. Estratégias, validade e confiabilidade. Ribeirāo Preto, 1987, 156 p. (Dissertaçāo de mestrado - Escola de Enfermagem de Ribeirāo Preto-USP). 
5 GONÇALVES, L.H.T. Desenvolvimento de um instrumento de pesquisa: medidas de atitude dos adultos em relação ao velho. Florianópolis, 1980, 81 p. (Tese professor titular - Departamento de Enfermagem da Universidade Federal de Santa Catarina).

6 LYNN, M.R. Reability estimates: use and disuse. Nurs Rev., New York, 34(4): 254-6, Jul./Ago. 1985.

7 NEVES, E.P. Vazios do conhecimento e sugestões de temática relevantes na área de enfermagem. In: SEMINÁRIO NACIONAL SOBRE O ENSINO DE PÓS-GRADUAÇÃO E PESQUISA EM ENFERMAGEM, 2, Brasília, 1982. p. 50-70.

8 NOTTER, L.E. Essencials of Nursing Research, New York, Springer Publishing, 1978.

9 NUNNALly, Jr., J.C. Introduction to Psychological Measurement. New York, Mc Graw Hill, 1970.

10 O'CONNELL, K.A. Nursing practice: a decade of research. In: CHASKA, N.L. The Nursing profession: a time to speak. New York, Mc Graw Hill, 1983, cap. 15. p. 183-201.

11. PINTO, A.V. Ciência e existéncia. Rio de Janeiro, Paz e Terra, 537 p.

12 POLIT, D.F. \& HUNGLER, B.P. Nursing research-principles and methods. Philadelphia, Lippincott, $1983.663 \mathrm{p}$.

13 TREECE, E.W. \& TREECE, J.W. Elements of research in nursing. Saint Lowis, Mosby, 1977. $349 \mathrm{p}$.

14 UBEDA, E.M.L. A educação para a saúde na escola: um estudo de algumas práticas de saúde desenvolvidas na pré-escola. Ribeirăo Preto, $1986.119 \mathrm{p}$. (Dissertaçăo de mestrado EERP-USP).

15 WILLIAMSON, M.Y. Research methodology and its application to nursing. New York, John Wiley, 1981 . 


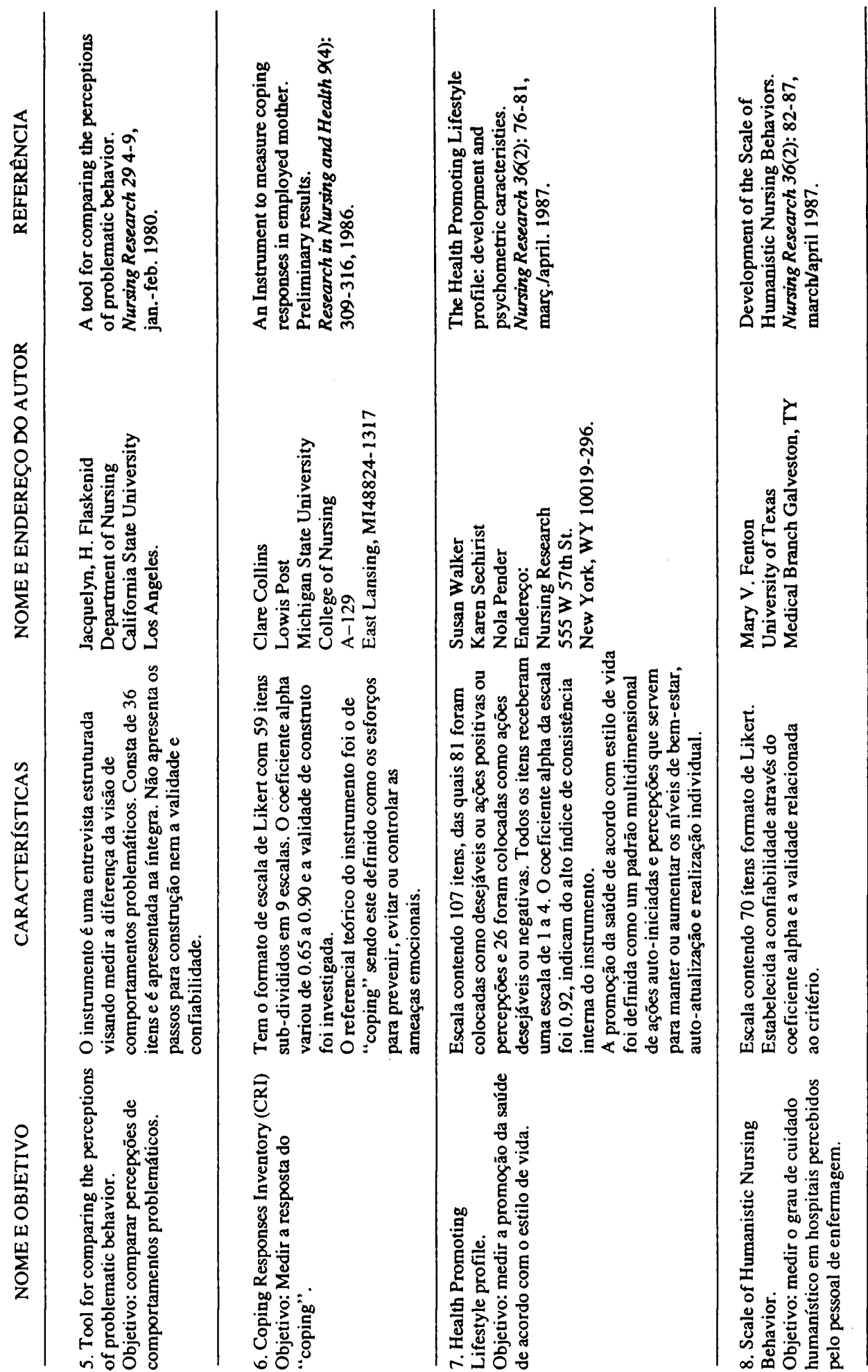




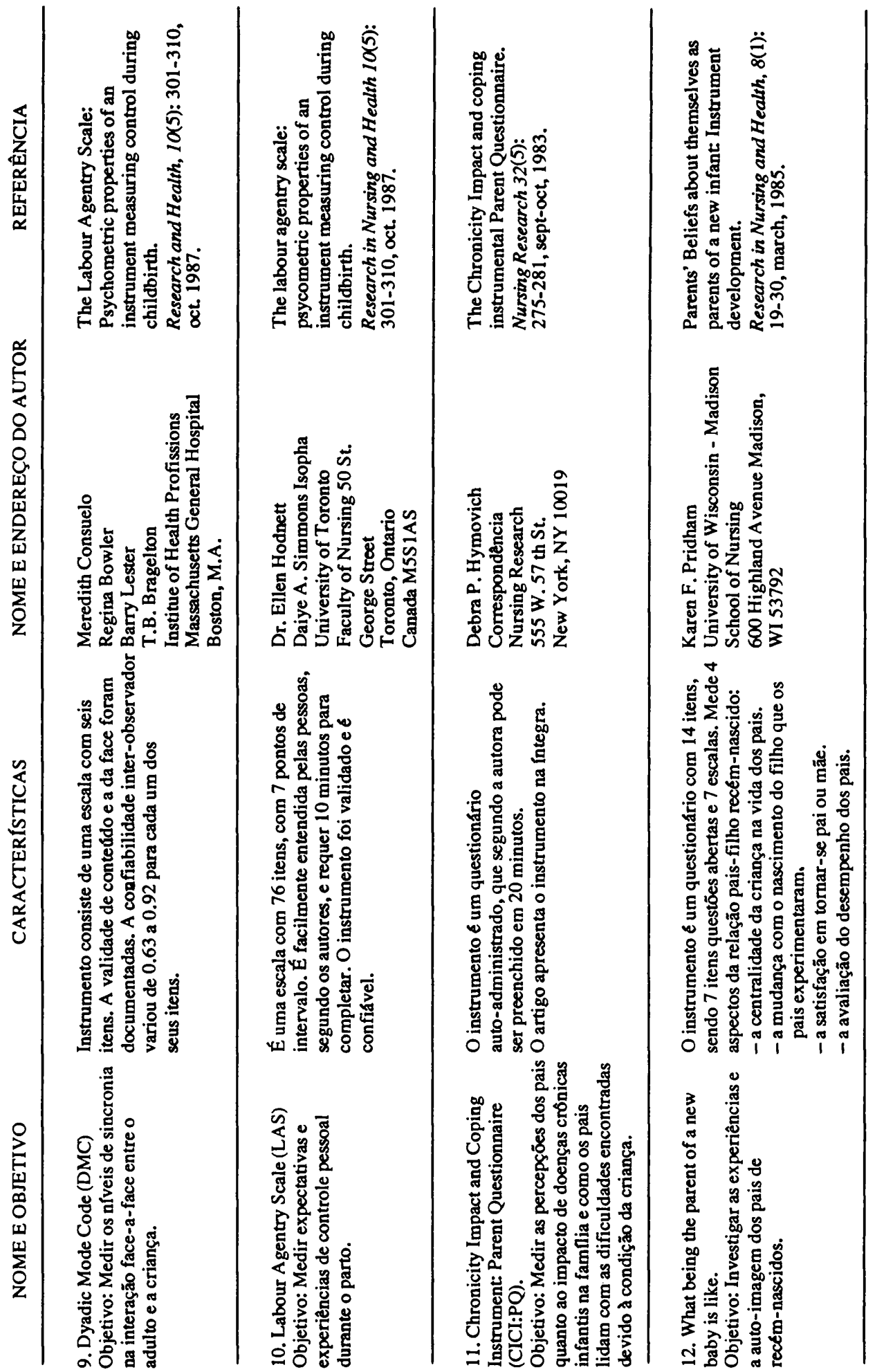




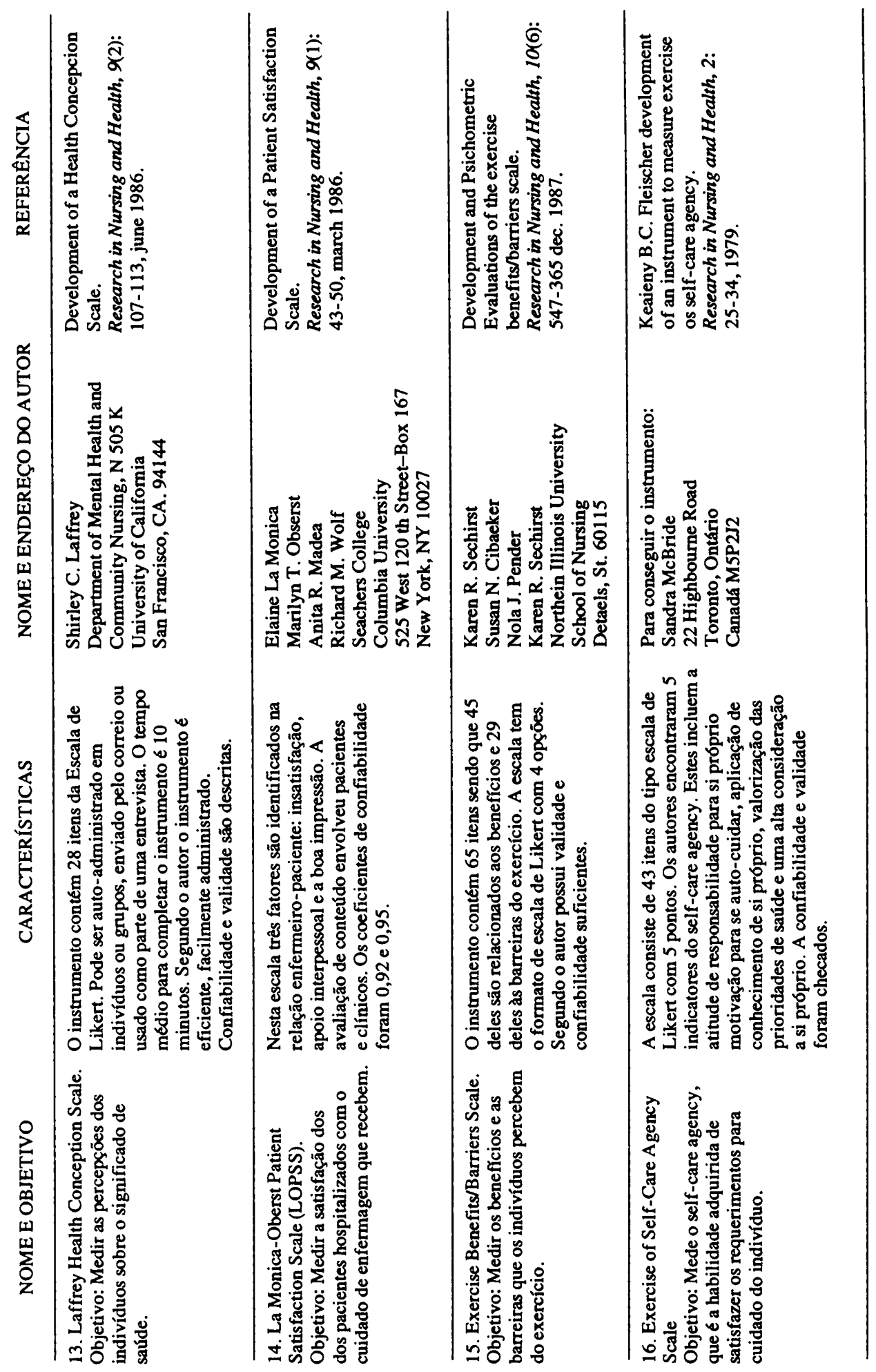




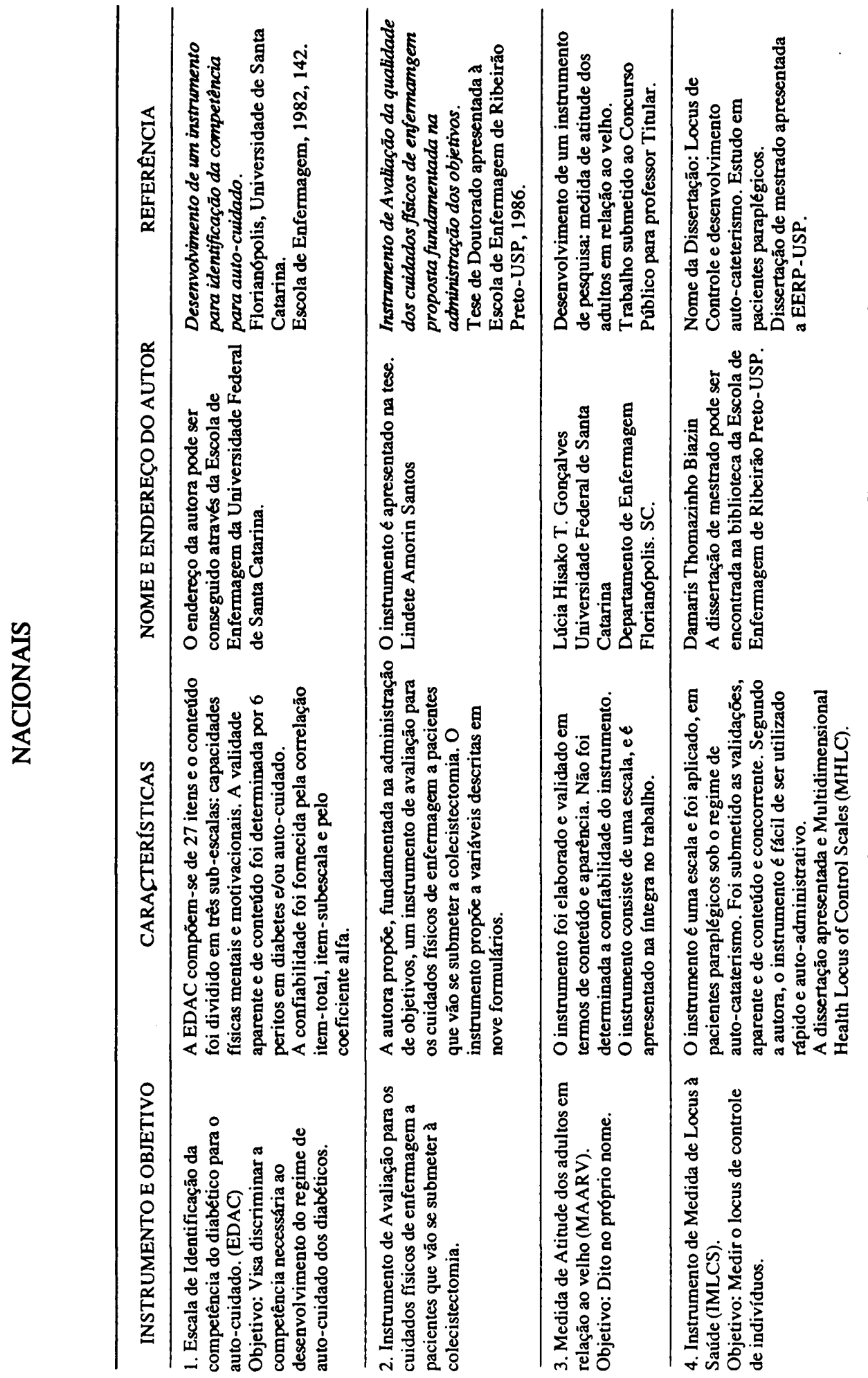

